OPEN ACCESS

Edited by:

Silvia Di Giacomo,

Sapienza University of Rome, Italy

Reviewed by:

Sadaf Jahan,

Majmaah University, Saudi Arabia

Rolf Teschke,

Hospital Hanau, Germany

${ }^{*}$ Correspondence:

Gianmarco Stati

statigianmarco@gmail.com

Specialty section: This article was submitted to Gastrointestinal and Hepatic

Pharmacology,

a section of the journal

Frontiers in Pharmacology

Received: 20 September 2021 Accepted: 18 October 2021

Published: 29 October 2021

Citation:

Stati G, Rossi F, Sancilio S, Basile M and Di Pietro $R$ (2021) Curcuma longa

Hepatotoxicity: A Baseless Accusation. Cases Assessed for

Causality Using RUCAM Method.

Front. Pharmacol. 12:780330

doi: 10.3389/fphar.2021.780330

\section{Curcuma longa Hepatotoxicity: A Baseless Accusation. Cases Assessed for Causality Using RUCAM Method}

\author{
Gianmarco Stati ${ }^{1 *}$, Francesco Rossi ${ }^{2,3}$, Silvia Sancilio ${ }^{1}$, Mariangela Basile ${ }^{1}$ and \\ Roberta Di Pietro ${ }^{1}$ \\ ${ }^{1}$ Department of Medicine and Ageing Sciences, G. d'Annunzio University of Chieti-Pescara, Chieti, Italy, ${ }^{2}$ Department of \\ Molecular Sciences and Nanosystems, Ca' Foscari University, Venice, Italy, ${ }^{3}$ Biophysics Group, Department of Physics and \\ Astronomy, University College London, London, United Kingdom
}

Curcuma longa is a perennial herb that belongs to the Zingiberaceae family. To date, literature includes more than 11.000 scientific articles describing all its beneficial properties. In the last 3 decades various surveys by the U.S. Food and Drug Administration (FDA) concluded that curcumin, the most active ingredient of the drug, is a "generally safe" compound with strong anti-oxidant effects. Curcuma longa was introduced in the daily diet by ayurvedic teachers due to its beneficial effects on health. Nonetheless, recently several reports, from the various global surveillance systems on the safety of plant products, pointed out cases of hepatotoxicity linked to consumption of food supplements containing powdered extract and preparations of Curcuma longa. The latest trend is the use of Curcuma longa as a weight-loss product in combination with piperine, which is used to increase its very low systemic bioavailability. Indeed, only $20 \mathrm{mg}$ piperine, one of the alkaloids found in black pepper (Piper nigrum), assumed at the same time with $2 \mathrm{~g}$ curcumin increased 20 -fold serum curcumin bioavailability. This combination of natural products is now present in several weight loss supplements containing Curcuma longa. The enhanced drug bioavailability caused by piperine is due to its potent inhibition of drug metabolism, being able to inhibit human P-glycoprotein and CYP3A4, while it interferes with UDP-glucose dehydrogenase and glucuronidation activities in liver. While only few cases of hepatotoxicity, assessed using Roussel Uclaf Causality Assessment Method (RUCAM) method, from prolonged intake of piperine and curcumin have been reported, it would be reasonable to speculate that the suspected toxicity of Curcuma longa could be due to the concomitant presence of piperine itself. Hence, not only there is the need of more basic research to understand the etiopathology of curcumin-related hepatotoxicity and of the combination curcumin-piperine, but human trials will be necessary to settle this dispute.

Keywords: Curcuma longa, curcumin, piperine, food supplement, hepatotoxicity, bioavailability enhancer, RUCAM 


\section{INTRODUCTION}

The aim of this short review is to trigger a more critical evaluation of scientific evidence existing in literature on potential hepatotoxicity of Curcuma longa. The revision of sources would be against the latest trend that blames this famous spice widely used for centuries. Curcuma longa has been used throughout human history for various purposes due to its wide range of biological activity (Sharifi-Rad et al., 2020). Curcumin was found to be the primary active component of the extract from the rhizome, known as turmeric. Curcumin is the ingredient responsible for the effects of turmeric as a drug in its long history of use in traditional Asian medicine for a wide variety of disorders.

The Compendium of Sushruta, the foundational text of Ayurveda dating to 250 BCE (Joshi et al., 2017), recommends an ointment containing turmeric, Curcuma longa powdered, to relieve the effects of poisoned food. It is not surprising, therefore, that curcumin is currently sold as a dietary supplement and that numerous clinical trials are ongoing to evaluate curcumin activity. In the last decade a large number of reports have been published on the beneficial effects of curcumin (Barchitta et al., 2019) and it has been repeatedly claimed that this natural product is efficient and safe for the prevention and treatment of several diseases (Abd El-Hack et al., 2021). Moreover, curcumin has been widely studied for its antioxidant, anti-inflammatory, and wound-healing effects (Menon and Sudheer, 2007; Shirban et al., 2021). This natural polyphenol is considered by some authors as a "wonder drug of life" (Gera et al., 2017) and it is categorized as a "generally recognized as safe" (GRAS) material, with a stable metabolism and low toxicity (Nelson et al., 2017).

Over recent years, food supplements containing Curcuma longa have been widely used by an increasing number of consumers and there is accumulating evidence that curcumin may not be so effective and safe. A number of reports have been issued that described the cases of highly probable drug-induced autoimmune hepatitis (DIAIH) ascribed to ingestion of Curcuma longa dietary supplement (Philips et al., 2020). That is, in contrast with the use, since ancient times, of Curcuma longa, as hepatoprotective (Rahmani et al., 2016; Tung et al., 2017; Peng et al., 2018) and for the treatment of digestive tract problems (Gera et al., 2017). Furthermore, in literature it is reported that curcumin may prevent oxidative stress-related liver disorder causing a series of metabolic reactions as i) decreasing the levels of alanine transaminase (ALT), aspartase transaminase (AST), and alkaline phosphatase (ALP). ii) It increases the expression of glutathione-S-transferase (GST), glutathione reductase (GR), glutathione peroxidase (GPx), superoxide dismutase (SOD) and catalase (CAT) while further iii) reducing NO production and inhibiting ROS formation (Farzaei et al., 2018).

The most common substance associated with Curcuma longa in its use as food supplement is piperine from Piper nigrum L. Black pepper (Piper nigrum L.) is the most used specie of pepper and it has found a worldwide use as a spice. Its history of use in traditional medicine is thousands of years old, being mentioned in Ayurvedic medicine treaties and in traditional Chinese medicine as early as the
250 BCE (Stojanović-Radić et al., 2019). The peculiar flavour of the piper family fruits, and its main pharmacological activity is given by piperine, a molecule of the alkaloid family. Between all the varieties of Piperaceae, Piper Nigrum L. contains the largest amount of piperine with certain cultivar reaching the $9 \%$ in weight of piperine content (Gorgani et al., 2017). Piperine structure consists of a benzodioxol ring connected to a chain with conjugated double bonds, which on the other extremity is attached to a piperidine moiety throughout a carbonylamide bond (Koul et al., 2000). The presence of a benzodioxol moiety in proximity with a conjugated system is a common feature of many bioactive molecules as psychoactive drugs (Almalki et al., 2020) and molecules able to inhibit the cytochrome P450 (Koul et al., 2000).

Piperine in particular plays a large role in traditional medicine for the treatment of flu-like symptoms and to increase appetite (Stojanović-Radić et al., 2019). In the last decade, a number of works have tested the medical properties of piperine as antioxidant (Vijayakumar et al., 2004), its effect on lipid metabolism (Du et al., 2020) and its anticancer capability (de Almeida et al., 2020). While the most important property of piperine, which is gaining the attention of the scientific community, is its ability to increase the bioavailability of drugs and other active molecules extracted by plants (Gorgani et al., 2017; Lee et al., 2018; Stojanović-Radić et al., 2019).

Piperine, as a component of black pepper, has been demonstrated to have a limited or no toxicity when assumed as a spice $(\approx 1 \mathrm{pg} / \mathrm{kg}$ per day). The European Food Safety Authority identified $5 \mathrm{mg} / \mathrm{kg}$ per day as the no observed adverse effects level (NOAEL) (Bolognesi et al., 2015; Burdock, 2016). On the metabolic level, piperine has been shown to have fundamental effects on p-glycoprotein and many enzyme systems, leading to enhancement of the absorption and bioavailability of several therapeutic drugs. Piperine's bioavailability enhancing property is also partly attributed to increased absorption as a result of its effect on the ultrastructure of intestinal brush border (Meghwal and Goswami, 2013).

\section{INFLUENCE OF PIPERINE ON CURCUMIN BIOAVAILABILITY}

Curcumin is a lipophilic polyphenol that is, nearly insoluble in water, with an oral bioavailability lower than $2 \%$. The molecule of curcumin, 1,7-bis(4-hydroxy-3- methoxyphenyl)-1,6-heptadiene3,5-dione (diferuloylmethane), when orally administrated, is quite stable in the acidic $\mathrm{pH}$ of the stomach (Wang et al., 1997), but it undergoes extensive first pass metabolism, is conjugated in the liver, and excreted in the feces. The main metabolites of this process are identified in curcumin glucuronide and curcumin sulfate (Ireson et al., 2001).

Its bioavailability can be enhanced by increasing its absorption and decreasing its metabolic clearance. The co-administration of curcumin with naturally occurring UDP-glucuronyl transferase (UGT) inhibitors, like piperine, improves curcumin bioavailability compared to curcumin alone (Shoba et al., 2007). Curcumin given alone at $2 \mathrm{~g} / \mathrm{kg}$ achieves a very low serum concentration over a period of $4 \mathrm{~h}$ while the co-administration of curcumin in the 


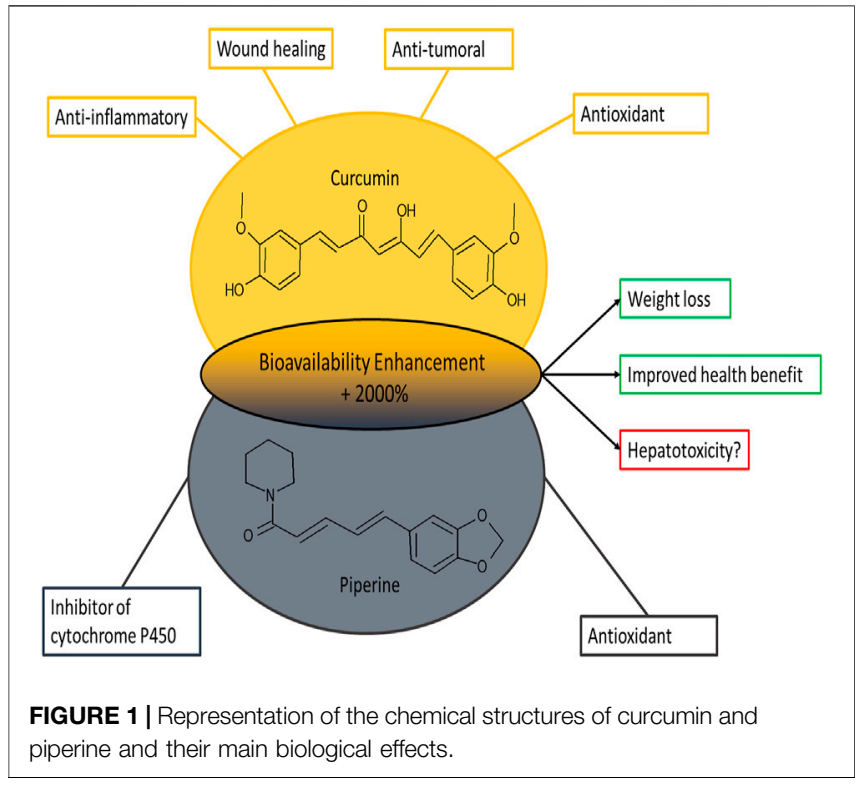

presence of $20 \mathrm{mg} / \mathrm{kg}$ of piperine increases its serum concentration for a short period of 1-2 $\mathrm{h}$ post drug intake (Chen et al., 2017). Piperine strongly influences a number of enzymatic biotransforming reactions in hepatic tissue, both in vitro and in vivo (Atal et al., 1985), acting as a non-specific inhibitor of drug metabolism which does not discriminate among the different forms of the cytochrome $\mathrm{P}_{450}$ (Dalvi and Dalvi, 1991).

In particular, piperine strongly inhibits hepatic microsomal aryl hydrocarbon hydroxylase (AHH) and UGT (Atal et al., 1985), leading to a significantly decreased clearance of the coadministered substance and a plasma half-life significantly increased. The overall increase in the bioavailability of curcumin by piperine is of the 2000\% (Shoba et al., 2007) (Figure 1).

Consuming turmeric and piperine separately is overall safe and it is even associated to a protective effect on cardiovascular mortality (Hashemian et al., 2019).

\section{DISCUSSION}

In recent years, numerous risk warnings related to products of natural origin have emerged. In particular, a series of reports on the possible hepatotoxicity of Curcuma longa, connected with the use of dietary supplements, have been published (Crijns et al., 2002; Costa et al., 2018; Lukefahr et al., 2018; Imam et al., 2019; Luber et al., 2019; Abdallah et al., 2020; Lee et al., 2020; Suhail et al., 2020; Lombardi et al., 2021). In the present article we have considered only reports who have applied Roussel Uclaf Causality Assessment Method (RUCAM) for causality assessment (Benichou et al., 1993; Danan and Benichou, 1993; Danan and
Teschke, 2016), since the weaknesses of LiverTox database approach include a case selection merely based on published case number and not on a strong causality assessment method such as RUCAM (Teschke and Danan, 2021). It is nowadays the suitable method applied in 81,856 DILI and HILI cases worldwide, according to (Teschke and Danan, 2020). The considered cases were judged as probable, supporting the causal relationship to the use of Curcuma longa containing supplements and herb-induced liver injury (HILI).

These reports have already caused a level of response from the regulatory bodies, for example, the European Medicine Agency (EMA) has revised its guideline in the September 25, 2018 excluding the use of Curcuma longa supplements in case of obstruction of the bile duct, cholangitis, liver disease, gallstones, and any other biliary diseases (European Medicines Agency, 2018). A change of the policy was a consequence of this report requiring from the January 1,2020 to add a specific warning on the label of food supplements containing curcumin, aimed at discouraging their use by people with liver disease, alterations in biliary function or with gallstones and, in case of concomitant drug intake, aimed at inviting them to consult the doctor (Ministero della Salute, 2019).

\section{CONCLUSION}

The overall limited number of cases worldwide, and the few toxicity studies available together with the preliminary determinations of regulatory organs seems to exclude the possibility of an intrinsic toxicity of curcumin. At the same time no solid evidence exists that the combination of curcumin and piperine could be the cause of hepatotoxicity. This seems to exonerate the spice which for millennia has been considered a panacea for all illnesses, indicating that the supposed Curcuma longa-related hepatotoxicity would be a baseless accusation. Overall, these type of food supplements should get the same level of attention from regulatory organs that is, given to drugs. Ideally, all the toxicity cases should be evaluated using a comparable method, as the updated RUCAM, and made available to the scientific community. For the specific case of curcumin, the number of cases reported is still too limited for definitive answer and only a more extensive clinical trial in presence of bioavailability enhancers could definitively settle this dispute.

\section{AUTHOR CONTRIBUTIONS}

GS wrote and conceptualized the paper. FR contributed in the writing and editing of the manuscript. SS and MB contributed to the literature search. RD contributed to vital revising. 


\section{REFERENCES}

Abd El-Hack, M. E., El-Saadony, M. T., Swelum, A. A., Arif, M., Abo Ghanima, M. M., Shukry, M., et al. (2021). Curcumin, the Active Substance of Turmeric: its Effects on Health and Ways to Improve its Bioavailability. J. Sci. Food Agric. 101, 5747-5762. doi:10.1002/jsfa.11372

Abdallah, M. A., Abdalla, A., Ellithi, M., Abdalla, A. O., Cunningham, A. G., Yeddi, A., et al. (2020). Turmeric-Associated Liver Injury. Am. J. Ther. 27, e642-e645. doi:10.1097/MJT.0000000000001025

Almalki, A. J., Clark, C. R., Abiedalla, Y., and DeRuiter, J. (2020). GC-MS Analysis of Methylenedioxybenzyl Analogues of the Serotonin Receptor Agonists 25XNBOMe Drugs. Forensic Chem. 21, 100284. doi:10.1016/J.FORC.2020.100284

Atal, C. K., Dubey, R. K., and Singh, J. (1985). Biochemical Basis of Enhanced Drug Bioavailability by Piperine: Evidence that Piperine Is a Potent Inhibitor of Drug Metabolism. J. Pharmacol. Exp. Ther. 232, 258-262. Available at: http://www. ncbi.nlm.nih.gov/pubmed/3917507 (Accessed September 17, 2021).

Barchitta, M., Maugeri, A., Favara, G., Magnano San Lio, R., Evola, G., Agodi, A., et al. (2019). Nutrition and Wound Healing: An Overview Focusing on the Beneficial Effects of Curcumin. Int. J. Mol. Sci. 20, 1119. doi:10.3390/ ijms20051119

Benichou, C., Danan, G., and Flahault, A. (1993). Causality Assessment of Adverse Reactions to Drugs--II. An Original Model for Validation of Drug Causality Assessment Methods: Case Reports with Positive Rechallenge. J. Clin. Epidemiol. 46, 1331-1336. doi:10.1016/0895-4356(93)90102-7

Bolognesi, C., Castle, L., Cravedi, J.-P., Engel, K.-H., Fowler, P., Franz, R., et al. (2015). Scientific Opinion on Flavouring Group Evaluation 86, Revision 2 (FGE.86Rev2): Consideration of Aliphatic and Arylalkyl Amines and Amides Evaluated by JECFA (65th Meeting). EFSA J. 13, 3998. doi:10.2903/ j.efsa.2015.3998

Burdock, G. A. (2016). Fenaroli's Handbook of Flavor Ingredients. Boca Ratón, FL: CRC Press. doi:10.1201/9781439847503

Chen, Z., Sun, D., Bi, X., Zeng, X., Luo, W., Cai, D., et al. (2017). Pharmacokinetic Based Study on "lagged Stimulation" of Curcumae Longae Rhizoma - Piper Nigrum Couplet in Their Main Active Components' Metabolism Using UPLCMS-MS. Phytomedicine 27, 15-22. doi:10.1016/j.phymed.2017.01.012

Costa, M. L., Rodrigues, J. A., Azevedo, J., Vasconcelos, V., Eiras, E., and Campos, M. G. (2018). Hepatotoxicity Induced by Paclitaxel Interaction with Turmeric in Association with a Microcystin from a Contaminated Dietary Supplement. Toxicon 150, 207-211. doi:10.1016/j.toxicon.2018.05.022

Crijns, A. P. G., de Smet, P. A. G. M., van den Heuvel, M., Schot, B. W., and Haagsma, E. B. (2002). Acute Hepatitis after Use of a Herbal Preparation with Greater Celandine (Chelidonium Majus), Ned. Tijdschr. Geneeskd., 146, 124-128. Available at: https://europepmc.org/article/med/11826672 (Accessed October 11, 2021).

Dalvi, R. R., and Dalvi, P. S. (1991). Comparison of the Effects of Piperine Administered Intragastrically and Intraperitoneally on the Liver and Liver Mixed-Function Oxidases in Rats. Drug Metabol. Drug Interact. 9, 23-30. doi:10.1515/DMDI.1991.9.1.23

Danan, G., and Benichou, C. (1993). Causality Assessment of Adverse Reactions to Drugs--I. A Novel Method Based on the Conclusions of International Consensus Meetings: Application to Drug-Induced Liver Injuries. J. Clin. Epidemiol. 46, 1323-1330. doi:10.1016/0895-4356(93)90101-6

Danan, G., and Teschke, R. (2016). RUCAM in Drug and Herb Induced Liver Injury: the Update. Int. J. Mol. Sci. 17 (1), 14. doi:10.3390/ijms17010014

de Almeida, G. C., Oliveira, L. F. S., Predes, D., Fokoue, H. H., Kuster, R. M., Oliveira, F. L., et al. (2020). Piperine Suppresses the Wnt/ $\beta$-Catenin Pathway and Has Anti-cancer Effects on Colorectal Cancer Cells. Sci. Rep. 10, 11681. doi:10.1038/s41598-020-68574-2

Du, Y., Chen, Y., Fu, X., Gu, J., Sun, Y., Zhang, Z., et al. (2020). Effects of Piperine on Lipid Metabolism in High-Fat Diet Induced Obese Mice. J. Funct. Foods 71, 104011. doi:10.1016/J.JFF.2020.104011

European Medicines Agency (2018). European Union Herbal Monograph on Curcuma Longa L., Rhizoma Final. Comm. Herb. Med. Prod. 44, 1-7. Available at: https://www. ema.europa.eu/en/documents/herbal-monograph/final-european-union-herbalmonograph-curcuma-longa-l-rhizoma-revision-1_en.pdf.

Farzaei, M. H., Zobeiri, M., Parvizi, F., El-Senduny, F. F., Marmouzi, I., CoyBarrera, E., et al. (2018). Curcumin in Liver Diseases: A Systematic Review of the Cellular Mechanisms of Oxidative Stress and Clinical Perspective. Nutrients 10, 855. doi:10.3390/nu10070855

Gera, M., Sharma, N., Ghosh, M., Huynh, D. L., Lee, S. J., Min, T., et al. (2017). Nanoformulations of Curcumin: an Emerging Paradigm for Improved Remedial Application. Oncotarget 8, 66680-66698. Available at:www. impactjournals.com/oncotarget/. doi:10.18632/oncotarget.19164

Gorgani, L., Mohammadi, M., Najafpour, G. D., and Nikzad, M. (2017). PiperineThe Bioactive Compound of Black Pepper: From Isolation to Medicinal Formulations. Compr. Rev. Food Sci. Food Saf. 16, 124-140. doi:10.1111/ $1541-4337.12246$

Hashemian, M., Poustchi, H., Murphy, G., Etemadi, A., Kamangar, F., Pourshams, A., et al. (2019). Turmeric, Pepper, Cinnamon, and Saffron Consumption and Mortality. Jaha 8. doi:10.1161/JAHA.119.012240

Imam, Z., Khasawneh, M., Jomaa, D., Iftikhar, H., and Sayedahmad, Z. (20192019). Drug Induced Liver Injury Attributed to a Curcumin Supplement. Case Rep. Gastrointest. Med. 2019, 6029403-6029404. doi:10.1155/2019/6029403

Ireson, C., Orr, S., Jones, D. J., Verschoyle, R., Lim, C. K., Luo, J. L., et al. (2001). Characterization of Metabolites of the Chemopreventive Agent Curcumin in Human and Rat Hepatocytes and in the Rat In Vivo, and Evaluation of Their Ability to Inhibit Phorbol Ester-Induced Prostaglandin E2 Production. Cancer Res. 61, 1058-1064.

Joshi, V. K., Joshi, A., and Dhiman, K. S. (2017). The Ayurvedic Pharmacopoeia of India, Development and Perspectives. J. Ethnopharmacol. 197, 32-38. doi:10.1016/J.JEP.2016.07.030

Koul, S., Koul, J. L., Taneja, S. C., Dhar, K. L., Jamwal, D. S., Singh, K., et al. (2000). Structure-activity Relationship of Piperine and its Synthetic Analogues for Their Inhibitory Potentials of Rat Hepatic Microsomal Constitutive and Inducible Cytochrome P450 Activities. Bioorg. Med. Chem. 8, 251-268. doi:10.1016/S0968-0896(99)00273-4

Lee, B. S., Bhatia, T., Chaya, C. T., Wen, R., Taira, M. T., and Lim, B. S. (2020). Autoimmune Hepatitis Associated with Turmeric Consumption. ACG Case Rep. J. 7, e00320. doi:10.14309/crj.0000000000000320

Lee, S. H., Kim, H. Y., Back, S. Y., and Han, H. K. (2018). Piperine-mediated Drug Interactions and Formulation Strategy for Piperine: Recent Advances and Future Perspectives. Expert Opin. Drug Metab. Toxicol. 14, 43-57. doi:10.1080/17425255.2018.1418854

Lombardi, N., Crescioli, G., Maggini, V., Ippoliti, I., Menniti-Ippolito, F., Gallo, E., et al. (2021). Acute Liver Injury Following Turmeric Use in Tuscany: An Analysis of the Italian Phytovigilance Database and Systematic Review of Case Reports. Br. J. Clin. Pharmacol. 87, 741-753. doi:10.1111/bcp.14460

Luber, R. P., Rentsch, C., Lontos, S., Pope, J. D., Aung, A. K., Schneider, H. G., et al. (2019). Turmeric Induced Liver Injury: A Report of Two Cases. Case Rep. Hepatol 2019, 6741213-6741214. doi:10.1155/2019/6741213

Lukefahr, A. L., McEvoy, S., Alfafara, C., and Funk, J. L. (2018). Drug-induced Autoimmune Hepatitis Associated with Turmeric Dietary Supplement Use. BMJ Case Rep. 2018, 1-7. doi:10.1136/bcr-2018-224611

Meghwal, M., and Goswami, T. K. (2013). Piper Nigrum and Piperine: An Update. Phytother Res. 27, 1121-1130. doi:10.1002/PTR.4972

Menon, V. P., and Sudheer, A. R. (2007). "Antioxidant and Anti-inflammatory Properties of Curcumin," in The Molecular Targets and Therapeutic Uses of Curcumin in Health and Disease (Boston, MA: Springer US), 105-125. doi:10.1007/978-0-387-46401-5_3

Ministero della Salute (2019). Food Supplements Containing Extract and Derivatives of Curcuma Longa. Available at: https://www.salute.gov.it/ portale/news/p3_2_1_1_1.jsp?

lingua $=$ italiano $\& \mathrm{menu}=$ notizie $\& \mathrm{p}=$ dalministero $\& \mathrm{id}=3842$

(Accessed September 17, 2021)

Nelson, K. M., Dahlin, J. L., Bisson, J., Graham, J., Pauli, G. F., and Walters, M. A. (2017). The Essential Medicinal Chemistry of Curcumin. J. Med. Chem. 60, 1620-1637. doi:10.1021/acs.jmedchem.6b00975

Peng, X., Dai, C., Liu, Q., Li, J., and Qiu, J. (2018). Curcumin Attenuates on Carbon Tetrachloride-Induced Acute Liver Injury in Mice via Modulation of the Nrf2/ HO-1 and TGF- $\beta 1 /$ Smad3 Pathway. Molecules 23, 215. doi:10.3390/ molecules 23010215

Philips, C. A., Ahamed, R., Rajesh, S., George, T., Mohanan, M., and Augustine, P. (2020). Comprehensive Review of Hepatotoxicity Associated with Traditional Indian Ayurvedic Herbs. World J. Hepatol. 12, 574-595. doi:10.4254/ WJH.V12.I9.574 
Rahmani, S., Asgary, S., Askari, G., Keshvari, M., Hatamipour, M., Feizi, A., et al. (2016). Treatment of Non-alcoholic Fatty Liver Disease with Curcumin: A Randomized Placebo-Controlled Trial. Phytother Res. 30, 1540-1548. doi:10.1002/PTR.5659

Sharifi-Rad, J., Rayess, Y. E., Rizk, A. A., Rizk, C., Zgheib, R., Zam, W., et al. (2020). Turmeric and its Major Compound Curcumin on Health: Bioactive Effects and Safety Profiles for Food, Pharmaceutical, Biotechnological and Medicinal Applications. Front. Pharmacol. 11, 01021-01023. doi:10.3389/ fphar.2020.01021

Shirban, F., Gharibpour, F., Ehteshami, A., Bagherniya, M., Sathyapalan, T., and Sahebkar, A. (2021). The Effects of Curcumin in the Treatment of Gingivitis: A Systematic Review of Clinical Trials. Adv. Exp. Med. Biol., 1291, 179-211. doi:10.1007/978-3-030-56153-6_11

Shoba, G., Joy, D., Joseph, T., Majeed, M., Rajendran, R., and Srinivas, P. S. (2007). Influence of Piperine on the Pharmacokinetics of Curcumin in Animals and Human Volunteers. Planta Med. 64, 353-356. doi:10.1055/S2006-957450

Stojanović-Radić, Z., Pejčić, M., Dimitrijević, M., Aleksić, A., V. Anil Kumar, N., Salehi, B., et al. (2019). Piperine-A Major Principle of Black Pepper: A Review of its Bioactivity and Studies. Appl. Sci. 9, 4270. doi:10.3390/app9204270

Suhail, F. K., Masood, U., Sharma, A., John, S., and Dhamoon, A. (2020). Turmeric Supplement Induced Hepatotoxicity: a Rare Complication of a Poorly Regulated Substance. Clin. Toxicol. (Phila) 58, 216-217. doi:10.1080/ 15563650.2019.1632882

Teschke, R., and Danan, G. (2021). Idiosyncratic Drug-Induced Liver Injury (DILI) and Herb-Induced Liver Injury (HILI): Diagnostic Algorithm Based on the Quantitative Roussel Uclaf Causality Assessment Method (RUCAM). Diagnostics (Basel) 11, 458. doi:10.3390/diagnostics11030458

Teschke, R., and Danan, G. (2020). Worldwide Use of RUCAM for Causality Assessment in 81,856 Idiosyncratic DILI and 14,029 HILI Cases Published
1993-Mid 2020: A Comprehensive Analysis. Medicines (Basel) 7, 62. doi:10.3390/medicines7100062

Tung, B. T., Hai, N. T., and Son, P. K. (2017). Hepatoprotective Effect of Phytosome Curcumin against Paracetamol-Induced Liver Toxicity in Mice. Braz. J. Pharm. Sci. 53, 1-13. doi:10.1590/s2175-97902017000116136

Vijayakumar, R. S., Surya, D., and Nalini, N. (2004). Antioxidant Efficacy of Black Pepper (Piper Nigrum L.) and Piperine in Rats with High Fat Diet Induced Oxidative Stress. Redox Rep. 9, 105-110. doi:10.1179/135100004225004742

Wang, Y. J., Pan, M. H., Cheng, A. L., Lin, L. I., Ho, Y. S., Hsieh, C. Y., et al. (1997). Stability of Curcumin in Buffer Solutions and Characterization of its Degradation Products. J. Pharm. Biomed. Anal. 15, 1867-1876. doi:10.1016/ S0731-7085(96)02024-9

Conflict of Interest: The authors declare that the research was conducted in the absence of any commercial or financial relationships that could be construed as a potential conflict of interest.

Publisher's Note: All claims expressed in this article are solely those of the authors and do not necessarily represent those of their affiliated organizations, or those of the publisher, the editors and the reviewers. Any product that may be evaluated in this article, or claim that may be made by its manufacturer, is not guaranteed or endorsed by the publisher.

Copyright (C) 2021 Stati, Rossi, Sancilio, Basile and Di Pietro. This is an open-access article distributed under the terms of the Creative Commons Attribution License (CC $B Y)$. The use, distribution or reproduction in other forums is permitted, provided the original author(s) and the copyright owner(s) are credited and that the original publication in this journal is cited, in accordance with accepted academic practice. No use, distribution or reproduction is permitted which does not comply with these terms. 